\section{Linfoma anaplásico de células grandes asociado a implantes mamarios diagnosticado mediante punción por aguja fina. Caso clínico}

\author{
CARLOS MISAD S. ${ }^{1}$, KENNETH WALKER B. ${ }^{1, a}$, \\ JOSÉ R. VALBUENA ${ }^{1}$, CLAUDIO GUERRA S. ${ }^{2}$, \\ MAURICIO CAMUS A. ${ }^{3}$, MAURICIO OCQUETEAU ${ }^{3}$, \\ SANDRA LOYOLA ${ }^{4, \mathrm{~b}}$, PABLO ZOROQUIAIN ${ }^{1, \mathrm{c}}$
}

\section{Anaplastic large cell lymphoma associated with breast implants, diagnosed by fine needle aspiration. Report of one case}

Breast implant-associated anaplastic large cell lymphoma (BIA-ALCL) is a rare type of non-Hodgkin T-cell lymphoma, recently defined in the 2016 World Health Organization (WHO) classification of lymphoid neoplasms. It occurs more commonly when textured implants are used and appears clinically as a late seroma. Cytologically, these lesions are composed of large atypical cells with pleomorphic nucleus and an immunophenotype positive for T cell markers and CD30, and negative for ALK1. We report a 56-years-old woman with breast implants who developed a periprosthetic seroma three years after surgery. A fine needle aspiration of the lesion was carried out. Cytology and the immunocytochemical study revealed cells compatible with BIA-ALCL. The flow cytometric study was negative. Excisional biopsy of the capsule was performed, observing that the neoplastic cells were confined to the inner surface of the capsule. Imaging studies did not find evidence of disseminated disease. The present case demonstrates the importance of the study of any late periprosthetic effusion, which can be performed using fine needle aspiration.

(Rev Med Chile 2020; 148: 1207-1212)

Key words: Breast Implants; Lymphoma, Non-Hodgkin; Pathology, Surgical.

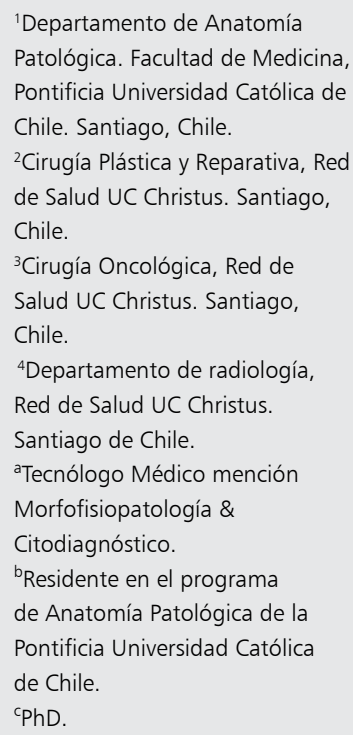

Trabajo no recibió financiamiento Los autores declaran no tener conflictos de interés.

Recibido el 1 de marzo de 2020, aceptado el 14 de agosto de 2020

Correspondencia a:

Dr. Pablo Zoroquiain. Marcoleta $377,10^{\circ}$ piso. Departamento de Anatomía Patológica, Pontificia Universidad Católica de Chile. Santiago, Chile. pzoro@ucchristus.cl.
E n el año 2016, la Organización Mundial de la Salud describió al linfoma anaplásico de $\checkmark$ células grandes asociado a implantes mamarios (LACG-AIM) como un subtipo de linfoma no Hodgkin de células $\mathrm{T}^{1}$ que surge en la cápsula del implante que puede haber sido colocado después de una reconstrucción por carcinoma o después de un aumento mamario estético ${ }^{2}$. La frecuencia del LACG-AIM es diferente según la población estudiada, siendo infrecuente en asiáticos. El número exacto de casos sigue siendo difícil de determinar, pero hasta el año 2019, se han reportado alrededor de 500 casos $^{3}$.
Se ha descrito que el LACG-AIM ocurre con más frecuencia cuando se han utilizado implantes con superficies texturizadas en lugar de aquellos con superficies lisas ${ }^{4}$.

Clínicamente, este linfoma se presenta con mayor frecuencia como un seroma de inicio tardío. Los seromas postoperatorios tempranos que se producen semanas a meses después de la cirugía no forman parte del espectro de la enfermedad de LACG-AIM ${ }^{5}$.

Presentamos un caso chileno de LACG-AIM diagnosticado mediante punción por aguja fina (PAF). 


\section{Presentación del caso}

Paciente de 56 años que se somete a una cirugía de aumento mamario mediante implantes de silicona texturizados. Se realiza controles con mamografía por 2 años resultando sin hallazgos significativos. A los 3 años y debido a un aumento progresivo del tamaño de la mama derecha, se realiza una tomografía computada de tórax en el que se observa una colección de líquido que rodea el implante mamario derecho, compatible con seroma. Debido a estos hallazgos, se realiza una resonancia magnética (Figuras $1 \mathrm{a}$ y b) confirmando un seroma periprotésico en la mama derecha por lo que se realiza una PAF guiada por ecografía.

Se extrajeron sólo $6 \mathrm{ml}$ de líquido seroso turbio que se dividió en dos, una parte para estudio citológico $(4 \mathrm{ml})$ y otra para citometría de flujo

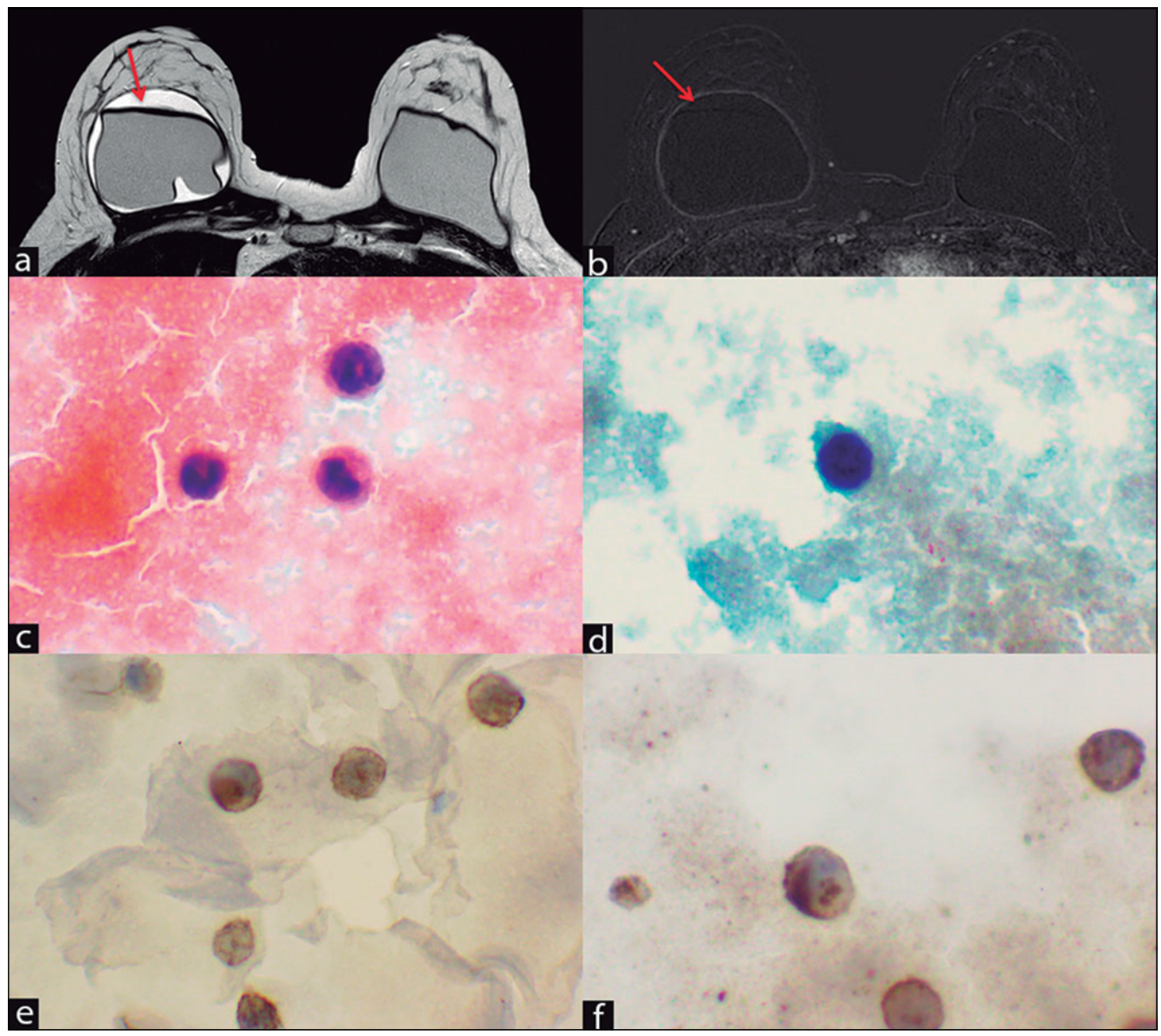

Figura 1. a. Resonancia magnética corte axial en T2 donde se observa moderada cantidad de líquido periprotésico en mama derecha sin evidencias de rotura protésica (flecha). b. Resonancia magnética. En estudio dinámico con contraste se observa intensificación uniforme de la cápsula periprotésica derecha sin masa asociada (flecha). c. Se identifican células grandes, con pleomorfismo nuclear, presencia de uno o múltiples nucléolos de variado tamaño, envoltura irregular y cromatina heterogénea (Tinción de Papanicolau, 20x). d. "Wreath Cells" o células guirnalda. Célula atípica con núcleo multilobular (Tinción de Papanicolau, 40x). e. Inmunocitoquímica positiva para CD30 (inmunomarcación con diaminobenzidina y contraste con hematoxilina de Mayer, 20x). f. Inmunocitoquímica positiva para CD45 (inmunomarcación con diaminobenzidina y contraste con hematoxilina de Mayer, 20x). 
(2 ml). Estudio por citometría de flujo demostró que $82 \%$ de las células eran viables y positivas para CD 45. El 18\% de ellas correspondieron a linfocitos de estirpe T con expresión normal de CD3, CD4, CD8 y CD5. El resto correspondieron a células de estirpe granulocítica.

El análisis citológico demostró escasas células atípicas grandes, redondas, con escaso citoplasma y núcleo hipercromático dispuestas de manera aisladas (Figura 1c). Se observaron también células con núcleo multilobulado (Figura 1d). Se realizaron estudios inmunocitoquímicos positivos para CD45 y CD30 (Figuras 1e y f) y negativos para CD3, CD20 y ALK-1(Figura 5), concluyéndose su compatibilidad con un LACG-AIM.

Por estos hallazgos, y 15 días posdiagnóstico citológico, se realizó remoción de los implantes con capsulectomía bilateral (Figuras $2 \mathrm{a}$ y $2 \mathrm{~b}$ ). El estudio del tejido capsular reveló tejido fibroconectivo infiltrado por una proliferación linfoide atípica de la zona interna del implante (Figura 2c). A mayor aumento, se observó que estas células son grandes, pleomórficas, con núcleos vesiculosos y escaso citoplasma (Figura 2d). Se realizó estudio inmunohistoquímico que mostró que las células atípicas eran positivas para CD43 (parcial), CD4, CD7 (parcial), CD30 y granzima $\mathrm{B}$. Los marcadores CD45, CD3, CD5, CD8 y CD20 resultaron negativos. Los hallazgos histológicos e inmunohistoquímicos fueron compatibles con LACG. Se realizó PET-CT 1 mes posoperatorio que no mostró adenopatías o enfermedad diseminada. $\mathrm{Al}$ seguimiento, a los 6 meses post-operatorio, no hay evidencias de recidiva.

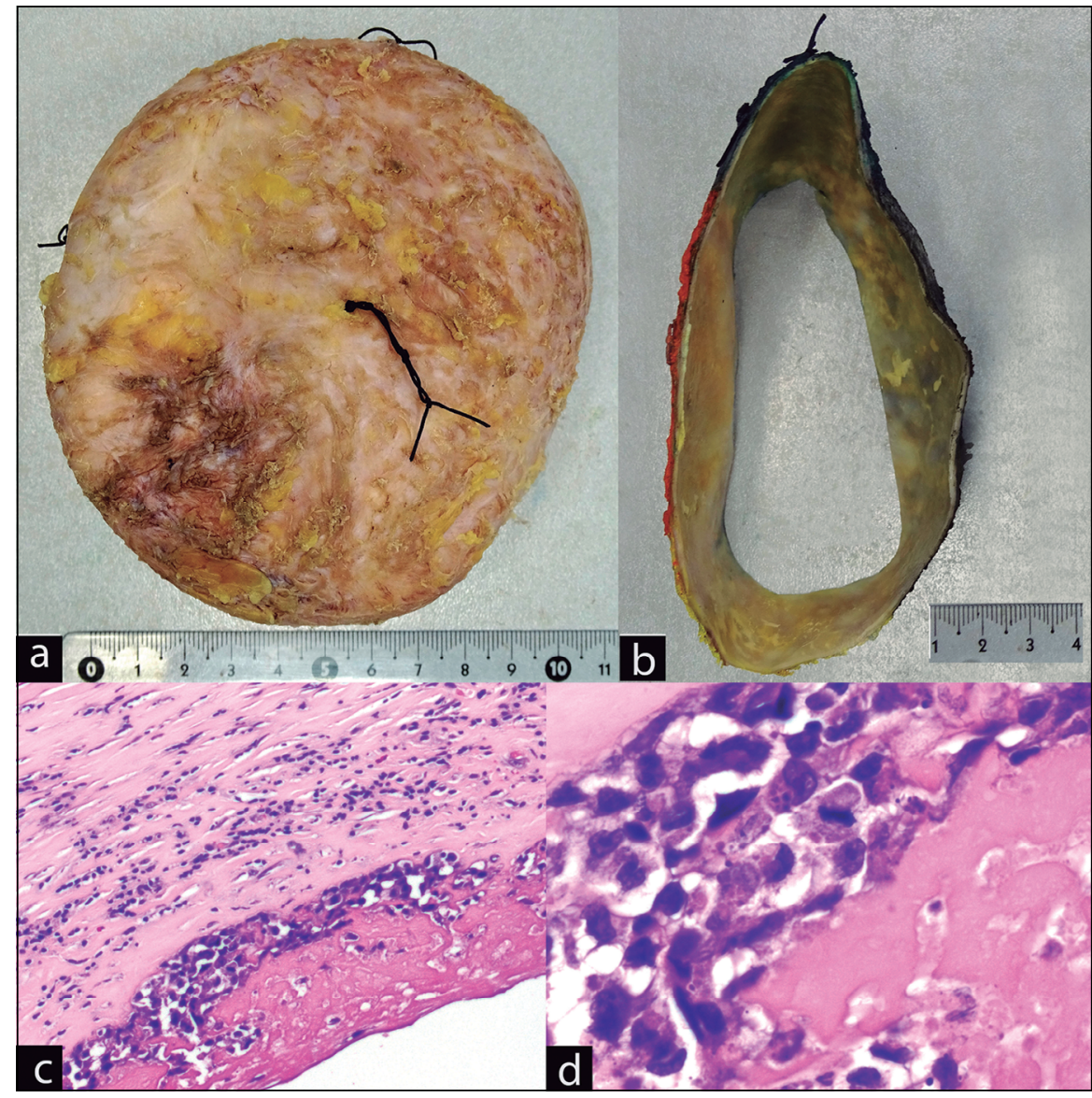

Figura 2. a. Pieza quirúrgica correspondiente a cápsula periprotésica con bordes quirúrgicos marcados con hilo de sutura. b. Corte transversal de la cápsula periprotésica con los bordes entintados para su posterior identificación microscópica. c. Tejido fibroconectivo correspondiente a cápsula del seroma, con infiltrado linfoide atípico (Hematoxilina-Eosina, 20x). d. A mayor aumento, linfocitos grandes, de núcleos vesiculosos y escasos citoplasma. Algunos de ellos necróticos (Hematoxilina-Eosina, 40x). 


\section{Discusión}

De acuerdo con lo revisado, este es el primer caso reportado de LACG-AIM diagnosticado mediante PAF en Chile. A nivel latinoamericano, existen 2 publicaciones una de Argentina y otra de México. Ambos casos se presentaron como seromas tardíos y fueron diagnosticados mediante PAF. Además, en el Encuentro Nacional de Cirugía Plástica de México de 2018, se presentaron 3 casos de México, 3 casos de Chile y 1 caso de Argenti$\mathrm{na}^{6-8}$. En Chile se habla en la prensa escrita de que habría a lo menos 6 casos, sin embargo, éstos no han sido, de acuerdo a lo revisado, comunicados.

El principal factor de riesgo son los implantes texturizados ${ }^{2}$. La silicona procesada contiene varios componentes que son potencialmente biológicamente activos como los grupos vinílicos residuales, que pueden llegar a los tejidos adyacentes ya sea por ruptura o infiltración tras la degradación de los im- plantes en el tiempo ${ }^{9}$. Finalmente, se ha propuesto que la silicona podría producir una estimulación crónica del sistema inmune, con posibles respuestas inmunes anormales mediadas por activación de linfocitos Th1 y Th7 que llevaría a la expresión de oncogenes como SATB1 y JunB que podrían producir la formación de neoplasias linfoides ${ }^{9,10}$.

Clínicamente, se presenta como una colección líquida (seroma periprotésico) o como una masa periprotésica. La forma más frecuente es la primera reconociéndose por un aumento brusco del volumen mamario ${ }^{11}$. La media de la edad son 51 años ${ }^{12}$.

Una vez sospechado, el estudio por imágenes es mandatorio. El estudio de seromas se debería complementar con punción por aguja fina guiada por ecografía. En el caso de estudio de masas o adenopatías el estudio es con biopsia ${ }^{13}$. Son pocos los casos reportados en donde haya información acerca del diagnóstico citológico realizado me-

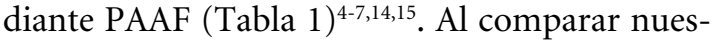

Tabla 1. Casos reportados de LACG-AIM con diagnostico citológico

\begin{tabular}{|c|c|c|c|c|c|c|}
\hline Publicación & Edad & $\begin{array}{l}\text { Intervalo desde } \\
\text { implante hasta } \\
\text { diagnóstico } \\
\text { (meses) }\end{array}$ & $\begin{array}{l}\text { Razón del } \\
\text { implante }\end{array}$ & $\begin{array}{l}\text { Tipo de } \\
\text { implante }\end{array}$ & $\begin{array}{l}\text { Inmunocito- } \\
\text { química }\end{array}$ & $\begin{array}{l}\text { Motivo de } \\
\text { consulta }\end{array}$ \\
\hline $\begin{array}{l}\text { Di Napoli et al } \\
\quad 1\end{array}$ & 47 & 72 & Reconstrucción & Texturizado & $\begin{array}{l}\text { CD30+, ALK-,CD8-, } \\
\text { CD3-, CD4+ }\end{array}$ & Seroma tardío \\
\hline 2 & 54 & 60 & Reconstrucción & Texturizado & $\begin{array}{c}\text { CD30+,ALK-,CD8-- } \\
\text { CD3-,CD4+ }\end{array}$ & Seroma tardio \\
\hline 3 & 72 & 72 & Cosmético & Texturizado & $\begin{array}{c}\text { CD30+, ALK-,CD8+, } \\
\text { CD4-, CD3- }\end{array}$ & Seroma tardio \\
\hline 4 & 49 & 96 & Reconstrucción & Texturizado & $\begin{array}{c}\text { CD30+, ALK-, CD8+, } \\
\text { CD4-, CD3+/- }\end{array}$ & Seroma tardio \\
\hline 5 & 35 & 48 & Cosmético & Silicona & $\begin{array}{c}\text { CD30+, ALK-, CD8- } \\
\text { CD4-, CD3+/- }\end{array}$ & Seroma tardio \\
\hline \multicolumn{7}{|l|}{ Wang et al } \\
\hline 6 & 75 & 60 & Reconstrucción & Texturizado & CD30+, ALK- & $\begin{array}{c}\text { Dolor, eritema, } \\
\text { aumento de volumen }\end{array}$ \\
\hline $\begin{array}{l}\text { Smith et al } \\
7\end{array}$ & 33 & 84 & Cosmético & Silicona & CD30+, ALK-, CD4+ & $\begin{array}{c}\text { Seroma } \\
\text { periprotésico }\end{array}$ \\
\hline $\begin{array}{l}\text { Ronchi et al } \\
8\end{array}$ & 43 & 24 & Cosmético & Silicona & $\begin{array}{c}\text { CD30+, ALK-, } \\
\text { CD4+,CD2+,CD3- }\end{array}$ & Seroma tardío \\
\hline $\begin{array}{l}\text { Torres-Rivero et al } \\
\quad 9\end{array}$ & 43 & 72 & Cosmético & Texturizado & $\begin{array}{c}\mathrm{CD} 30+, \mathrm{ALK}-, \mathrm{CD} 45+ \\
\mathrm{CD} 3+, \mathrm{CD} 20-\end{array}$ & $\begin{array}{l}\text { Dolor, edema y } \\
\text { galactorrea }\end{array}$ \\
\hline $\begin{array}{l}\text { Mariel García et al } \\
10\end{array}$ & 33 & 72 & Cosmético & Texturizado & $\begin{array}{c}\mathrm{CD} 30+, \mathrm{ALK}-, \mathrm{CD} 3+ \\
\text { CD20- }\end{array}$ & $\begin{array}{l}\text { Aumento de } \\
\text { volumen }\end{array}$ \\
\hline
\end{tabular}


tros resultados con éstos, vemos que todos tienen marcador CD30 positivo y ALK negativo, pero en el nuestro el marcador de linfocito T resultó negativo.

La citología podría confundirse con el de un contenido de quiste corriente con abundantes macrófagos de tamaño variable. Sin embargo, se observa además una población de células de núcleos multilobulados, que pueden estar dispuestos en herradura. Estas células son llamadas "Hallmark Cells"5. El diagnóstico de LACG-AIM debe apoyarse de un perfil inmunohistoquímico positivo para CD30 y negativo para $\mathrm{ALK}^{16}$. Los antígenos de células $\mathrm{T}$ se expresan de manera variable, siendo el más común CD4 $(80 \%$ a $84 \%$ por ciento de los casos), CD43 (80\%-88\%) y en menor medida, CD3 (30\%-46\%), CD45 (36\%) y CD2 (30\%). La expresión de CD5, CD7, CD8 o CD15 es rara ${ }^{17}$.

El manejo de LACG-AIM incluye la remoción del implante, la cápsula, cualquier masa y adenopatías. En las muestras extirpadas el análisis histopatológico es mandatorio. Las células neoplásicas se disponen en la cara interna de la cápsula, pero hay que ponerle atención a los cúmulos celulares, ya que estos pueden infiltrar la cápsula, tejido blando circundante o el parénquima mamario ${ }^{16}$. Mientras el linfoma permanezca confinado en el espacio del seroma y el borde esté negativo, el pronóstico es excelente pudiendo sólo ser tratados con cirugía ${ }^{18}$.

En conclusión, se ha visto un aumento del LACG-AIM asociado a las cirugías con implantes, sobre todo en aquellas en las que se utilizan implantes con superficie rugosa. Es importante considerar esta entidad dentro del diagnóstico diferencial de todo paciente con seromas periprotésicos tardíos o con tumoración capsular. El estudio citológico del derrame periprotésico es una herramienta útil para el diagnóstico precoz de LACG-AIM, ya que aquellos casos sin diseminación tienen un pronóstico favorable en comparación con aquellos donde hay enfermedad extramamaria.

\section{Referencias}

1. Swerdlow SH, Campo E, Pileri SA, Harris NL, Stein H, Siebert R, et al. The 2016 revision of the World Health Organization classification of lymphoid neoplasms. Blood 2016; 127 (20): 2375-90.

2. Kricheldorff J, Fallenberg EM, Solbach C, Gerber-Schä- fer C, Rancsó C, von Fritschen U. Breast implantassociated lymphoma. Dtsch Aerzteblatt Online; Disponible en: https://www.aerzteblatt.de/10.3238/ arztebl.2018.0628 [Consultado el 28 de diciembre de 2019].

3. Fitzal F, Turner SD, Kenner L. Is breast implant-associated anaplastic large cell lymphoma a hazard of breast implant surgery? Open Biol 2019; 9 (4): 190006.

4. Wang JS, Degeorge BR, Showalter SL, Morgan RF, Lin KY. Anaplastic Large Cell Lymphoma Associated with Double-Lumen Breast Implants: A Case Report and Review of the Literature. Plast Surg Case Stud 2016; 2 (2): 20-2.

5. Di Napoli A, Pepe G, Giarnieri E, Cippitelli C, Bonifacino A, Mattei M, et al. Cytological diagnostic features of late breast implant seromas: From reactive to anaplastic large cell lymphoma. Tripodo C, editor. PLoS ONE. 2017; 12 (7): e0181097.

6. Torres-Rivero C, Ramos-Gallardo G, Nambo-Lucio MJ, Vaquero-Pérez MM. Primer caso en México y América Latina de linfoma anaplásico de células gigantes en paciente con implantes mamarios. Cir Plast Iberolatinoam 2016, 42 (2): 175-80.

7. García AM, Uriburu JL, Candás GB, Isetta J, Cogorno L, Sigal SM, et al. Linfoma anaplásico de células grandes (lacg) asociado a implante mamario. Reporte de un caso y revisión de la literatura. Rev Argent Mastología 2017; 36 (132): 9-18.

8. Ramos-Gallardo G, Cuenca-Pardo J, Cárdenas-Camarena L, Durán-Vega H, Rodríguez-Olivares E, Bayter-Marin JE, et al. Is Latin America Ready to Identify Anaplastic Large Cell Lymphoma in Breast Implants Patients? Regional Encounter During the National Plastic Surgery Meeting in Cancun, Mexico. Aesthetic Plast Surg 2018; 42 (5): 1421-8.

9. Rupani A, Frame JD, Kamel D. Lymphomas Associated with Breast Implants: A Review of the Literature. Aesthet Surg J 2015; 35 (5): 533-44.

10. Kadin ME, Deva A, Xu H, Morgan J, Khare P, MacLeod RAF, et al. Biomarkers Provide Clues to Early Events in the Pathogenesis of Breast Implant-Associated Anaplastic Large Cell Lymphoma. Aesthet Surg J 2016; 36 (7): 773-81.

11. Clemens MW, Nava MB, Rocco N, Miranda RN. Understanding rare adverse sequelae of breast implants: anaplastic large-cell lymphoma, late seromas, and double capsules. Gland Surg 2017; 6 (2): 169-84.

12. Johnson L, O'Donoghue JM, McLean N, Turton P, Khan AA, Turner SD, et al. Breast implant associated anaplastic large cell lymphoma: The UK experience. Recommendations on its management and implications 
for informed consent. Eur J Surg Oncol EJSO 2017; 43 (8): 1393-401.

13. van Dorp M, T'Kindt J, Mertens M, Colpaert SDM. Novel Technique for Sampling of Breast Implant-associated Seroma in Anaplastic Large Cell Lymphoma: Plast Reconstr Surg - Glob Open 2016; 4 (4): e688.

14. Smith TJ, Ramsaroop R. Breast implant related Anaplastic Large Cell Lymphoma presenting as late onset peri-implant effusion. The Breast. 2012; 21 (1): 102-4.

15. Ronchi A, Montella M, Argenzio V, Lucia A, De Renzo A, Alfano R, et al. Diagnosis of anaplastic large cell lymphoma on late peri-implant breast seroma: Management of cytological sample by an integrated approach.
Cytopathology 2018; 29 (3): 294-9.

16. Barbé E, Boer M, Jong D. A practical cytological approach to the diagnosis of breast-implant associated anaplastic large cell lymphoma. Cytopathology 2019; 30 (4): 363-9.

17. Xu J, Wei S. Breast Implant-Associated Anaplastic Large Cell Lymphoma: Review of a Distinct Clinicopathologic Entity. Arch Pathol Lab Med 2014; 138 (6): 842-6.

18. Miranda RN, Aladily TN, Prince HM, Kanagal-Shamanna $\mathrm{R}$, de Jong $\mathrm{D}$, Fayad LE, et al. Breast Implant-Associated Anaplastic Large-Cell Lymphoma: Long-Term Follow-Up of 60 Patients. J Clin Oncol 2014; 32 (2): 114-20. 